\title{
Metastatic Hepatocellular Carcinoma to the Nasal Cavity: A Case Report and Review of the Literature
} Chih-Yi Liu' ${ }^{1 *}$ Liang-Che Chang ${ }^{2}$ and Shih-Wei Yang ${ }^{3}$

1Department of Pathology, Cathay General Hospital, Sijhih, Taipei, Taiwan 2Department of Pathology, Chang Gung Memorial Hospital, Keelung, Taiwan

3Department of Otolaryngology Head and Neck Surgery, Chang Gung Memorial Hospital, Keelung, Taiwan

\begin{abstract}
Hepatocellular carcinoma is a common primary hepatic malignancy in the Asian countries. The prognosis of metastatic hepatocellular carcinoma is very poor if left untreated. Despite the rare occurrence, sinonasal metastasis of hepatocellular carcinoma usually implies an advanced disease stage with dismal outcome. Herein we present a case of metastatic hepatocellular carcinoma involving the nasal cavity. This 55-year-old male patient refused standard therapy after the diagnosis of hepatocellular carcinoma. After a palliative excision for the nasal tumor, the patient died of multiple metastatic diseases. A special consideration should be given to such unusual manifestations of extrahepatic metastases. Promptly clinical evaluation and appropriate intervention for symptomatic palliation are necessary for these terminally ill patients.
\end{abstract}

Keywords: Hepatocellular carcinoma; Metastasis; Nasal cavity

\section{Introduction}

Metastatic tumors are rarely encountered in the nasal cavity. They comprise less than $1 \%$ of the nasal malignancies. Reviewing the literature, the most common primary tumor site is the kidney (more than $60 \%$ ), while most of the remainder arise in the lung, breast, urogenital tract, gastrointestinal tract and thyroid gland [4]. Sinonasal region is an unusual site for metastatic hepatocellular carcinoma (HCC), and there are only two reported cases with solely nasal metastases $[2,6]$. Herein we report an additional case of metastatic HCC to the nasal cavity. Our case demonstrates disseminated diseases after the appearance of nasal tumor. The rapid deterioration is probably due to the refusal of standard therapy for HCC. Because such unusual extrahepatic manifestations always lead to a dismal prognosis, the clinicians should be aware of the importance of appropriate clinical staging and palliative treatment modalities.

\section{Case Report}

A 55-year-old male patient had hepatitis B-related, decompensated liver cirrhosis diagnosed in 2005. At that time, abdominal sonography and CT scan revealed a tumor mass in the segment 7 of liver, associated with venous thrombi involving the portal vein and inferior vena cava. The subsequent histopathological examination of liver biopsy demonstrated hepatocellular carcinoma. The patient refused standard therapy and took herb drug for eight months. The patient's general condition became deteriorated. Multiple metastases in the bilateral lungs and the mediastinal lymph nodes were noticed in April 2006. One month later, the patient visited our otorhinolaryngologic outpatient department and complaint of progressively growing right nasal mass found in the past three weeks. Intermittent episodes of epistaxis are also noted for two months. Physical examination revealed a brown to black polypoid tumor mass bulging out from the vestibule and vault of right nasal cavity (Figure 1). The surface of tumor is granular and encrusted. CT scan showed a $2.7 \times 2.2 \times 2.0 \mathrm{~cm}$ tumor nodule in the right nasal cavity, invading the nasal ala and eroding the underlying maxillary bone. The patient underwent excisional biopsy of the tumor mass to relief symptoms.

Pathological examination of the nasal tumor revealed polygonal neoplastic cells growing in broad trabeculae or solid sheets with intervening sinusoid-like vascular spaces (Figure 2). The tumor predominantly invaded the subepithelial stroma with mucosal ulceration and hemorrhage. Occasional vascular space permeation was noticed as well. A panel of immunohistochemical study showed tumor cells staining positive for Hep Par 1 (hepatocyte paraffin 1) (Figure 3A) and CK (cytokeratin). Cytoplasmic staining for TTF-1 (thyroid transcription factor-1) was also observed (Figure 3B), while AFP (alpha fetoprotein), EMA (epithelial membrane antigen) and CK7 revealed negative results. The above histopathological features confirmed of the diagnosis of hepatocellular carcinoma metastasizing to the nasal cavity.

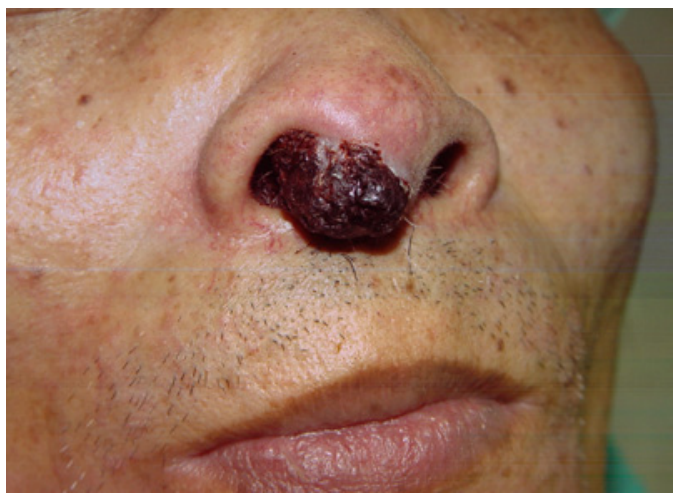

Figure 1: A brown to black, mulberry-like mass bulging out from right nasal cavity..

*Corresponding author: Chih-Yi Liu MD, Department of Pathology, Cathay General Hospital, Sijhih, Taipei, Taiwan, Add: \#2, Lane 59, Chien-Cheng Road, Sijhih City, Taipei County, Taiwan, Tel: 886-2-26482121 ext.3741; Fax: 886-282370777; E-mail: cyl1124@gmail.com

Received January 29, 2011; Accepted March 02, 2011; Published March 09, 2011

Citation: Liu CY, Chang LC, Yang SW (2011) Metastatic Hepatocellular Carcinoma to the Nasal Cavity: A Case Report and Review of the Literature. J Cancer Sci Ther 3: 081-083. doi:10.4172/1948-5956.1000064

Copyright: (c) 2011 Liu CY, et al. This is an open-access article distributed under the terms of the Creative Commons Attribution License, which permits unrestricted use, distribution, and reproduction in any medium, provided the original author and source are credited. 
Citation: Liu CY, Chang LC, Yang SW (2011) Metastatic Hepatocellular Carcinoma to the Nasal Cavity: A Case Report and Review of the Literature. J Cancer Sci Ther 3: 081-083. doi:10.4172/1948-5956.1000064

The patient was discharged in a stable condition. Unfortunately, progressive limb weakness developed in June 2006, and subsequent image study depicted brain metastasis with intracranial hemorrhage. The patient died of multiple metastatic diseases, two months after the appearance of nasal mass.

\section{Discussion}

Hepatocellular carcinoma (HCC) is one of the most frequently occurring malignancies in Asian populations. The high incidence in Asia is due to the high prevalence of chronic viral hepatitis, particularly chronic hepatitis B. In Taiwan, the annual incidence of HCC is about 10

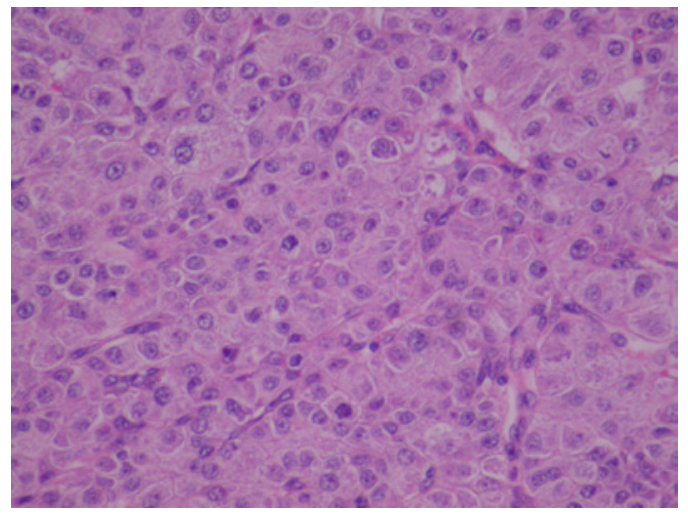

Figure 2: Microscopic examination shows features of metastatic hepatocellular carcinoma with trabeculae lined by polygonal tumor cells. (H\&E, original magnification $\times 400$ ).
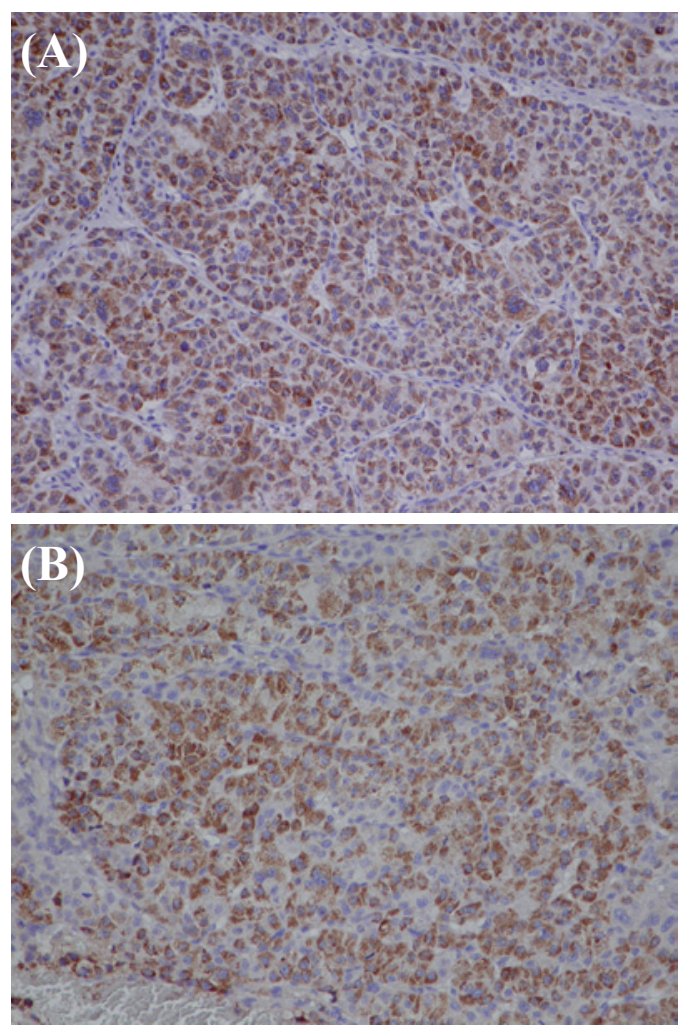

Figure 3: Tumor cells reveal positive immunohistochemical stain for Hep Par 1 (A), and cytoplasmic staining for TTF-1 (B) (Immunoperoxidase, original magnification $\times 200$ ). to 25 cases per 100,000 populations [3]. Owing to the early surveillance program for HCC high-risk groups and the longer survival periods, the incidence of metastatic HCC increases with time [9]. The bone and lung are the most common metastatic sites for HCC, while oro-maxillofacial metastasis is indeed an unusual presentation.

The typical symptoms of sinonasal metastatic tumor include nasal obstruction, epistaxis, pain, local swelling and exophthalmos, which are indistinguishable from those of the primary nasal neoplasm [2]. The most frequent site of primary origin is the kidney, followed by lung, breast, urogenital tract, gastrointestinal tract and thyroid gland [3]. Secondary sinonasal deposits from the liver are rarely encountered. Reviewing the English literatures, there were only fourteen cases of metastatic HCCs involving the paranasal sinus and the nose $[1,3,4,5,6,8]$. More than one half of (nine out of fourteen) cases were reported from Asian countries, including Japan (5 cases), Taiwan ( 1 case), Hong Kong ( 1 case), Singapore ( 1 case) and Korea (1 case) $[1,3,4,5,8]$. It raises a concern for the possibility of metastatic HCCs presenting as sinonasal tumors, especially among the Asian countries showing a higher incidence. In addition, the symptoms of metastases may be the initial manifestation of malignancies. Okada et al indicated that 17 (59\%) of the 29 reviewed cases developed metastatic HCCs in the oro-maxillo-facial region before the discovery of liver tumors [5]. Because of the poor prognosis and fatal clinical outcome, metastatic tumors should always be included in the differential diagnoses of rapidly growing sinonasal lesions [9]. Detailed clinical history and thoroughly histopathological assessment are necessary for a firm diagnosis [7]. As in our case, the elaborated immunohistochemical profile with positive reactivity for hepatocyte markers distinguishes metastatic HCC from other nasal malignancies.

In the present case, the metastatic tumor invades the nasal vestibule, vault and ala, with repeated episodes of hemorrhage. As revealed by image study, there are multiple metastases in the both lungs and the mediastinal lymph nodes, just before the tumor metastasizes to the nose and sequentially the brain. The metastatic route of HCC appears to be through lymphogenous or hematogenous spread such as caval venous system, thence to the lung and bone $[2,3,4,5]$. For those patients with liver cirrhosis, it has been proposed that the retrograde flow of the prevertebral and vertebral venous plexus may be the other pathway of extrahepatic spread $[3,4,5]$. On the other hand, multiple extrahepatic metastases of HCC could occur via the vertebral venous plexus even without lung deposits. In the 29 cases of oro-maxillo-facial metastatic HCCs reviewed by Okada et al, at least 11 patients did not have lung lesions at autopsy or by diagnostic imaging [5]. Sim et al described a case of isolated sphenoid sinus metastasis in the absence of other demonstrable secondaries [8]. Therefore, it may be more difficult to detect asymptomatic or occult metastatic diseases in such rare extrahepatic locations.

The occurrence of sinonasal metastatic HCC always leads to a poor clinical outcome. The expected life span after the appearance of sinonasal lesions is limited, ranging from 6 weeks to 21 months $[1,3,4,5,6]$. Sinonasal metastasis is usually indicative of widespread tumor dissemination, while further curative treatment seems to be unattainable. Prompt surgical excision or electrocauterization for the bulky tumor may play a role in symptomatic palliation, such as nasal obstruction and epistaxis [9] Otherwise, some patients were successfully treated by transcatheter arterial embolization for the control of local bleeding.

In the endemic areas of viral hepatitis, HCC is one of the most frequently occurring malignancies. Our case revealed the existence of 
Citation: Liu CY, Chang LC, Yang SW (2011) Metastatic Hepatocellular Carcinoma to the Nasal Cavity: A Case Report and Review of the Literature. J Cancer Sci Ther 3: 081-083. doi:10.4172/1948-5956.1000064

intranasal metastatic HCC, a rare anatomical location for extrahepatic metastasis. The clinicians and the pathologists should always be aware of such unusual presentation owing to the poor prognosis and the possibility of rapid deterioration.

\section{References}

1. Aung TH, Po YC, Wong WK (2002) Hepatocellular carcinoma with metastasis to the skull base, pituitary gland, sphenoid sinus, and cavernous sinus. Hong Kong Med J 8: 48-51.

2. Lee HM, Kang HJ, Lee $\mathrm{SH}$ (2005) Metastatic renal cell carcinoma presenting as epistaxis. Eur Arch Otorhinolaryngol 262: 69-71.

3. Lin CD, Cheng KS, Tsai CH, Chen CL, Tsai MH (2002) Metastatic hepatocellular carcinoma in the nasal septum: report of a case. J Formos Med Assoc 101: 715-718

4. Matsuda H, Tanigaki Y, Yoshida T, Matsuda R, Tsukuda M (2006) A case of metastatic hepatocellular carcinoma in the nasal cavity. Eur Arch Otorhinolaryngol 263: 305-307.

5. Okada H, Kamino Y, Shimo M, Kitamura E, Katoh T, et al. (2003) Metastatic hepatocellular carcinoma of the maxillary sinus: a rare autopsy case without lung metastasis and a review. Int J Oral Maxillofac Surg 32: 97-100.

6. Patankar T, Prasad S, Patni S, James P (1998) Hypervascular nasal metastasis from hepatocellular carcinoma. Indian J Gastroenterol 17: 111-112.

7. Pires FR, Sagarra R, Correa ME, Pereira CM, Vargas PA, et al. (2004) Ora metastasis of a hepatocellular carcinoma. Oral Surg Oral Med Oral Pathol Oral Radiol Endod 97: 359-368.

8. Sim RS, Tan HK (1994) A case of metastatic hepatocellular carcinoma of the sphenoid sinus. J Laryngol Otol 108: 503-504.

9. Wang HY, Su CY, Lin JW, Chien CY (2004) Base of the tongue metastatic cancer from hepatocellular carcinoma: a case report. Eur Arch Otorhinolaryngol 261: 531-533. 